\title{
A pooled testing strategy for identifying SARS-CoV-2 at low prevalence
}

https://doi.org/10.1038/s41586-020-2885-5

Received: 13 May 2020

Accepted: 12 October 2020

Published online: 21 October 2020

Check for updates

\author{
Leon Mutesa ${ }^{1,2}$, Pacifique Ndishimye ${ }^{2,3}$, Yvan Butera ${ }^{1,2}$, Jacob Souopguii ${ }^{1,2,4}$, Annette Uwineza ${ }^{1,2}$, \\ Robert Rutayisire ${ }^{1,2}$, Ella Larissa Ndoricimpaye ${ }^{2}$, Emile Musoni ${ }^{2}$, Nadine Rujeni' ${ }^{2}$, \\ Thierry Nyatanyi ${ }^{2}$, Edouard Ntagwabira ${ }^{2}$, Muhammed Semakula ${ }^{2}$, Clarisse Musanabaganwa ${ }^{2}$, \\ Daniel Nyamwasa ${ }^{2}$, Maurice Ndashimye ${ }^{2,3}$, Eva Ujeneza ${ }^{3}$, Ivan Emile Mwikarago ${ }^{2}$, \\ Claude Mambo Muvunyi ${ }^{2}$, Jean Baptiste Mazarati ${ }^{2}$, Sabin Nsanzimana ${ }^{2}$, Neil Turok ${ }^{3,5,6} \bowtie$ \& \\ Wilfred Ndifon ${ }^{3 凶}$
}

Suppressing infections of severe acute respiratory syndrome coronavirus 2 (SARS-CoV-2) will probably require the rapid identification and isolation of individuals infected with the virus on an ongoing basis. Reverse-transcription polymerase chain reaction (RT-PCR) tests are accurate but costly, which makes the regular testing of every individual expensive. These costs are a challenge for all countries around the world, but particularly for low-to-middle-income countries. Cost reductions can be achieved by pooling (or combining) subsamples and testing them in groups ${ }^{1-7}$. A balance must be struck between increasing the group size and retaining test sensitivity, as sample dilution increases the likelihood of false-negative test results for individuals with a low viral load in the sampled region at the time of the test ${ }^{8}$. Similarly, minimizing the number of tests to reduce costs must be balanced against minimizing the time that testing takes, to reduce the spread of the infection. Here we propose an algorithm for pooling subsamples based on the geometry of a hypercube that, at low prevalence, accurately identifies individuals infected with SARS-CoV-2 in a small number of tests and few rounds of testing. We discuss the optimal group size and explain why, given the highly infectious nature of the disease, largely parallel searches are preferred. We report proof-of-concept experiments in which a positive subsample was detected even when diluted 100 -fold with negative subsamples (compared with 30-48-fold dilutions described in previous studies ${ }^{9-11}$ ). We quantify the loss of sensitivity due to dilution and discuss how it may be mitigated by the frequent re-testing of groups, for example. With the use of these methods, the cost of mass testing could be reduced by a large factor. At low prevalence, the costs decrease in rough proportion to the prevalence. Field trials of our approach are under way in Rwanda and South Africa. The use of group testing on a massive scale to monitor infection rates closely and continually in a population, along with the rapid and effective isolation of people with SARS-CoV-2 infections, provides a promising pathway towards the long-term control of coronavirus disease 2019 (COVID-19).
COVID-19 represents a major threat to global health. Rapidly identifying and isolating individuals with SARS-CoV-2 infections is one of the most important available strategies for containing the virus. However, each diagnostic test ${ }^{12}$ for the SARS-CoV-2 virus costs US $\$ 50-100$. Therefore, testing individuals regularly-which may be required to eliminate the virus-is expensive. The costs are unaffordable for most low-income countries, which have limited available resources for large-scale SARS-CoV-2 testing. It is therefore important to investigate whether there are more-efficient ways to identify those individuals infected with the virus.

The first step in testing-swab collection-is labour-intensive but does not require expensive chemicals or equipment. It may therefore be feasible to collect swabs regularly from everyone. The next step involves RT-PCR machines ${ }^{13}$. These require expensive chemical reagents, which are currently in short supply, as well as skilled personnel. To reduce the cost, we need to minimize the total number of tests. The speed of

${ }^{1}$ Centre for Human Genetics, College of Medicine and Health Sciences, University of Rwanda, Kigali, Rwanda. ${ }^{2}$ Rwanda Joint Task Force COVID-19, Rwanda Biomedical Centre, Ministry of

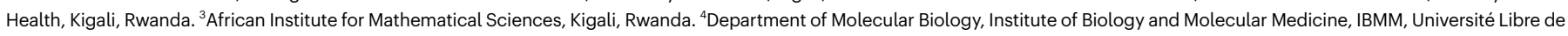
Bruxelles, Gosselies, Belgium. ${ }^{5}$ School of Physics and Astronomy, University of Edinburgh, Edinburgh, UK. ${ }^{6}$ Perimeter Institute for Theoretical Physics, Waterloo, Ontario, Canada.

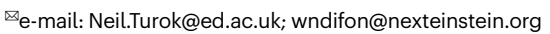


testing is also a key concern because SARS-CoV-2 is so infectious. Each RT-PCR test takes several hours in the laboratory, time during which the virus can spread ${ }^{14}$.

To identify individuals infected with SARS-CoV-2, the naive approach is to test everyone separately-that is, to perform one test per person. However, at low prevalence it is far more efficient to pool (or combine) samples and test these sample pools together. This idea of group testing was proposed by Dorfman in $1943^{1}$. At low viral prevalence $p$, Dorfman's algorithm reduces the number of tests per person to $\approx 2 \sqrt{ } p$ (Supplementary Information I). The algorithm that we present is more efficient, as it requires only $\approx e p \ln (1 / p)$ tests per person at low $p$, where $e=2.718 \ldots$ is Euler's number (Supplementary Information II). As an example, a survey of private residential households in England and Wales, released on 4 September 2020 by the Office of National Statistics ${ }^{15-17}$, estimated a prevalence of $p=0.05 \%$ (95\% confidence interval, $0.04-0.07 \%$ ). For $p=0.05 \%$, Dorfman's algorithm offers a 22 -fold cost reduction whereas ours offers a 100-fold cost reduction. The main obstacle to achieving these large cost savings is the number of samples that can be pooled without compromising detection. Here we present proof-of-concept experiments that show that one positive sample in a pool of a hundred samples can still be reliably detected. We also discuss how the pool size could be further increased to obtain the full benefits of group testing at low prevalence. If larger pool sizes and the associated cost reductions can be achieved, group testing may provide an affordable pathway to the long-term control of SARS-CoV-2.

In this paper, our focus is on population screening and not on protocols for use with at-risk groups or in clinical settings. The prevalence is typically much higher among individuals in at-risk groups or among individuals who present themselves for testing. For example, in the week ending 2 September $2020,0.6 \%$ of the tests performed in hospitals in England were positive ${ }^{18}$, which suggests a prevalence that is an order of magnitude greater than that in the wider population, quoted above. In addition, in clinical settings the overriding concern should be to test the individual patient as quickly and accurately as possible. In most situations, that means performing an individual test. We are not suggesting the use of group testing as a strategy for testing patients in clinics, especially those with symptoms.

With this caveat, there are many potential applications of our method-for example, to screen sports teams whose players and staff must be tested regularly. A prominent rugby team in South Africa is now trialling our method. Early results indicate cost savings of more than an order of magnitude, with the successful detection of positive samples in groups of 81 . Other applications include screening of staff and residents in care homes or pre-flight screening of passengers for commercial flights. The Government of Rwanda has adopted group testing as a national strategy and all air passengers are required to undergo a group test locally. This has helped to revive tourism in the country. Regular screening of university halls of residence, laboratories or departments could similarly enable safer in-person interactions. There is also potential for combining group testing with cheaper multiplex RT-PCR tests ${ }^{19}$. The combination could reduce the costs of population-wide screening by more than two orders of magnitude compared with current methods.

Group-testing algorithms generally require more than one round of testing. In Dorfman's algorithm, a first round of group tests is followed by a second round in which each member of every positive group is tested individually. Our algorithm involves a similar first round of group tests, although with a larger group size. Positive groups proceed to a second round of 'slice tests', which usually suffices to identify all individuals who are infected, without any need for individual tests. Occasionally one and, very rarely, more than one additional round of slice tests are required. We compare our approach with other approaches in detail in the Supplementary Information. There are adaptive algorithms that require fewer tests but more rounds of testing, during which time viral prevalence can grow. Such searches are disfavoured at low

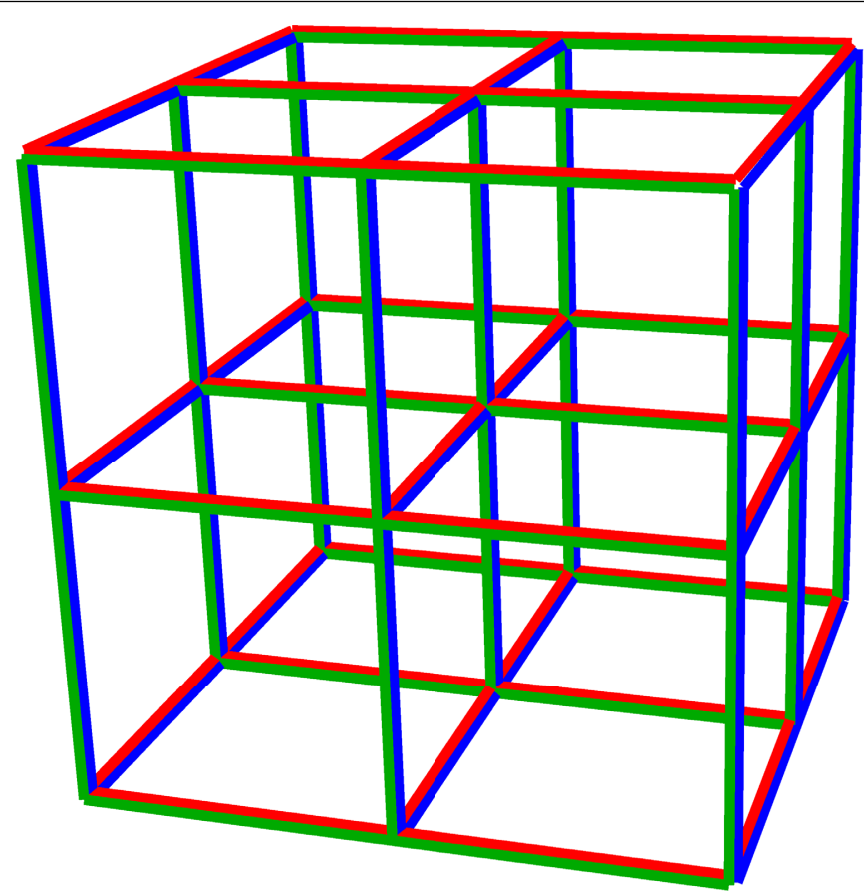

Fig. 1 Subsample pooling in the hypercube algorithm, shown here for $\boldsymbol{D}=\boldsymbol{L}=\mathbf{3}$ and $\boldsymbol{N}=\mathbf{2 7}=\mathbf{3}^{\mathbf{3}}$. Each vertex represents an individual. The hypercube is sliced into $L$ slices, in each of the $D$ principal directions. Samples from $N / L$ individuals are pooled into a sample for each slice. For this example, the three sets of slices are shown in blue, red and green. If, among the $N$ individuals being screened, only one is infected, tests on each set of slices identify their coordinate in that direction. Thus, in this example, only nine tests uniquely identify them. As the viral prevalence $p$ decreases, the optimal group size $N$, the dimension $D$ and the efficiency gain increase.

prevalence (see 'Largely parallel searches are preferred'). There are also non-adaptive algorithms that require only one round of testing ${ }^{4-7,11}$. Although these algorithms appear attractive, they have disadvantages compared with our algorithm-for example, a higher failure rate (Supplementary Information sections III, IX). In our approach, the first round of tests-which are performed on groups-provides a valuable 'sanity check' on the viral prevalence in the population, before the second round of more-numerous slice tests is performed. More generally, group tests can provide a highly efficient means of tracking the viral prevalence in various populations in real time (Supplementary Information VIII).

Group testing is most obviously effective when none of the group members is infected: just one test suffices to clear everyone. Our algorithm takes full advantage of this powerful result. In the first round of tests, subsamples from all group members are pooled and tested together. For our algorithm, the optimal group size is $N \approx 0.35 / p$. The expected number of group members who are infected is 0.35 and a group will test negative more than $70 \%$ of the time. Groups that test positive are passed on to the first round of slice tests, which we describe next.

\section{When one member of a group is infected}

Consider the case in which only one member of the group is infected. The idea behind our algorithm is geometrical: the group of individuals to be tested is represented by a set of $N$ points on a cubic lattice in $D$ dimensions, organized in the form of a hypercube with $L$ points on a side (Fig. 1), so that

$$
L^{D}=N
$$


Instead of directly testing the samples taken from every individual, we first divide each of them into $D$ equal subsamples. These $D N$ subsamples are recombined as follows. Slice the hypercube into $L$ planar slices, perpendicular to one of the principal directions on the lattice. Form such a set of slices in each of the $D$ principal directions and pool the $L^{D-1}=N / L$ subsamples that correspond to each slice. Altogether, $D L$ slices are tested in parallel, in each round of slice tests. If there is one individual who is infected, then one slice out of the $L$ slices in each of the $D$ directions will yield a positive result. That slice indicates the coordinate of the individual in the corresponding principal direction.

Therefore, the total number of tests required to find the individual who is infected is

$$
D L=D N^{1 / D}
$$

where we used equation (1). Treating $D$ as a continuous variable, the right side of equation (2) diverges at both small and large $D$, possessing a minimum at

$$
D=\ln (N),
$$

corresponding to $L=e$ and a total of $e \ln (N)$ tests. In reality, $D$ and $L$ must be integers, but using $L=3$ achieves almost the same efficiency (in the total number of tests, $e$ is replaced with $3 / \ln (3) \approx 2.73$, which is less than $0.5 \%$ larger, whereas using $L=2$ or 4 gives $2 / \ln (2)=4 / \ln (4) \approx 2.89$, which is more than $5 \%$ larger). With no further constraint, finding one person who is infected in a population of a million-using $L=3$-requires only 39 tests, performed in one round of testing. To understand this calculation, note that $3^{13}>10^{6}$; therefore, a hypercube of side $L=3$ with dimension $D=13$ contains more than a million points. A round of slice tests on this hypercube consists of $D L=13 \times 3=39$ slice tests.

\section{Proof of concept}

In real-time PCR tests, the target RNA molecules are reverse-transcribed into DNA, which is replicated exponentially until it can be detected using fluorescence. In a perfectly efficient test, the number of DNA molecules doubles in every PCR cycle. The test is extremely sensitive: fewer than 10 molecules of viral RNA are sufficient for a positive test result $^{13}$. A nasopharyngeal swab taken in the first 5 days of symptoms yields, on average, around $2 \times 10^{5}$ viral RNA molecules per millilitre ${ }^{20}$. Asymptomatic individuals who are infectious appear to have similar viral loads ${ }^{21}$. In the usual testing protocol, just $5 \mu$ l of the solution, containing on average around 1,000 RNA molecules, is included in the mix that is then analysed by PCR. Samples taken at earlier or later stages of infection, or in younger patients whose antibodies have suppressed the virus, have fewer virus particles present. In practice, this reduced number of virus particles is thought to be the most important cause of testing error, taking the form of false-negative test results ${ }^{17,22,23}$. In pooled testing, positive subsamples are diluted with negative subsamples. Dilution by 100 -fold, for example, means that on average only around 10 RNA molecules are likely to be present in the RT-PCR test. In principle, this should still be sufficient to yield a positive result.

As a proof of concept, using oropharyngeal swab specimens collected during COVID-19 surveillance in Rwanda, we investigated whether known positive specimens still tested positive after they were diluted 20-,50- or 100-fold through pooling with negative specimens (Methods). We used a RT-PCR test that targets the $N$ and orf $1 a b$ genes of SARS-CoV-2, a combination that is used routinely for diagnostic screening for SARS-CoV-2 infections in Rwanda. The standard protocol is to consider a test positive if PCR amplification produces an above-background fluorescence signal for both target genes at a PCR cycle number-that is, a cycle-threshold $\left(C_{\mathrm{t}}\right)$ value- of $C_{\mathrm{t}} \leq 40$. Our key finding is that typical positive specimens can still be detected even after dilution by up to a 100-fold (Fig. 2). Previously published
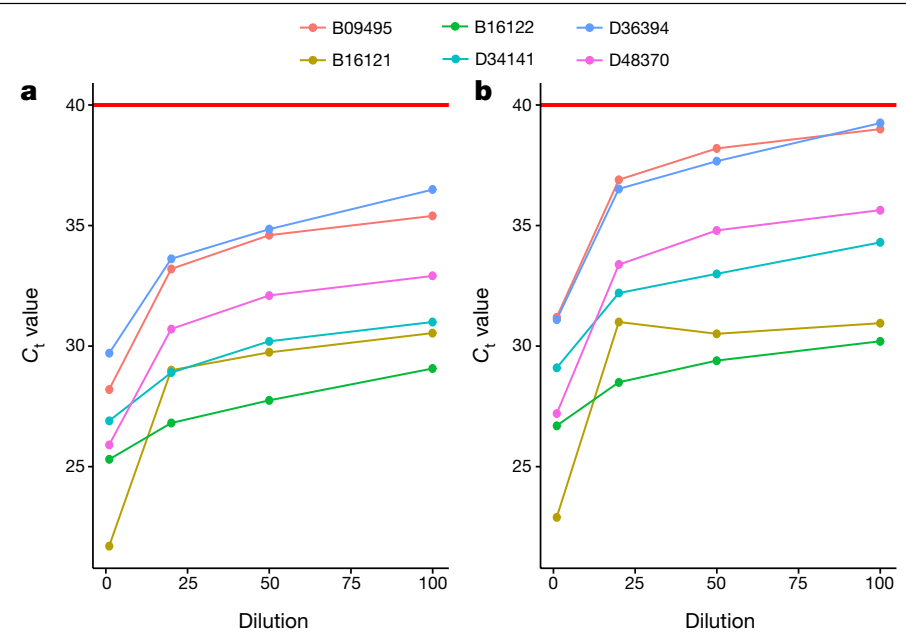

Fig. 2 | Positive specimens are detected after a 100-fold dilution. a, b, Each of six typical SARS-CoV-2-positive specimens was diluted through pooling with 19,49 or 99 negative specimens. A $C_{\mathrm{t}}$ value (that is, the PCR cycle at which the fluorescence signal generated by a specimen exceeds the baseline signal) was determined for each pool through RT-PCR amplification of the $N$ (a) and orf1ab (b) genes of SARS-CoV-2. For each gene, the $C_{\mathrm{t}}$ values are plotted against the dilution factor. The red horizontal lines indicate the $C_{\mathrm{t}}$ value (40) at or below which a specimen is considered positive. All $C_{\mathrm{t}}$ curves stay below the red lines even if the positive specimens are diluted 100-fold (Extended Data Fig. 1 and Extended Data Table 2).

experiments ${ }^{9-11}$ have demonstrated detection after 30 -, 32- and 48 -fold dilution. As a consistency check, we determined the change in the $C_{\mathrm{t}}$ value $\left(\Delta C_{\mathrm{t}}\right)$ when going from a 50 - to a 100 -fold dilution. As noted above, a positive sample diluted 100 -fold in principle requires one more cycle of PCR amplification than when diluted 50-fold to achieve the same fluorescence signal, implying $\Delta C_{\mathrm{t}} \approx 1.0$. Consistent with this expectation, we find $\Delta C_{t} \approx 1.0 \pm 0.15$ (mean \pm s.d.) for the $N$ gene and $\Delta C_{\mathrm{t}} \approx 1.1 \pm 0.14$ for the orflabgene. The changes in $C_{\mathrm{t}}$ values for other dilutions are also consistent with this interpretation.

We estimated the postdilution sensitivities by combining (technically, convolving) the probability distribution for predilution $C_{\mathrm{t}}$ values for positive samples (Extended Data Table 3) with the probability distribution for the increase in $\Delta C_{\mathrm{t}}$ as inferred above. Treating both distributions as Gaussian, the distribution of postdilution $C_{\mathrm{t}}$ values is also Gaussian, with the mean given by the sum of the means and the variance given by the sum of the variances. In this way, we estimated that a 40-cycle PCR test targeting the SARS-CoV-2 N (or orf1ab) gene, respectively, has postdilution sensitivities for 20-, 50- and 100-fold dilutions of $91 \%, 88 \%$ and $85 \%$ (or $85 \%, 81 \%$ and $77 \%$, respectively). We have confirmed these estimates using two additional datasets. First, we used an independent sample of $C_{\mathrm{t}}$ values for 107 positive specimens collected in Rwanda using tests that targeted the same two genes. Second, we reanalysed a published dataset of 26 positive specimens from a recent study of pooled testing for SARS-CoV-2 in Israel $^{10}$, in which a single gene (the $E$ gene) was targeted. All three datasets gave broadly consistent results.

The positive samples that are most likely to be missed because of dilution are those with the highest $C_{\mathrm{t}}$ values before dilution-that is, those with the lowest viral load. The individuals concerned are likely to be the least infectious ${ }^{24,25}$. Conversely, those individuals-whether symptomatic or asymptomatic-whose samples have the lowest $C_{\mathrm{t}}$ values, which are the least affected by sample dilution, are the most important to detect as they are likely to be the most infectious. Nevertheless, it is important to consider ways in which the loss in sensitivity due to dilution might be mitigated. The most obvious is to re-test sufficiently often (say, every 3 days) to ensure a test occurs in the period of highest viral 
abundance, for any individual infected with SARS-CoV-2. Group tests involve the greatest degree of dilution in our method, but they are also the cheapest testing stage to repeat frequently and to thereby mitigate sensitivity loss. Likewise, one could increase the number of PCR cycles to 44 , the maximum used in the previous study ${ }^{10}$. Similarly, the volume of the sample used in the RT-PCR test can be increased from $5 \mu \mathrm{l}$ to $10 \mu \mathrm{l}$, reducing the $C_{\mathrm{t}}$ value by one (this is done in the laboratory that we are working with in South Africa). Furthermore, the viral concentration in the pooled sample might be increased by physical or chemical methods such as ultracentrifugation or precipitation. Finally, PCR machines might be re-engineered to enable larger sample volumes to be tested. All of these possibilities are worth exploring.

\section{When more than one member of a group is infected}

So far, we have assumed that only one member of the group is infected. We must also consider what happens when two, three or more members of a group are infected. We will have to discover that number in the course of the slice tests. A feature of group testing is that the first round of group tests, which are relatively few in number, allows us to conveniently update our knowledge of the viral prevalence $p$, before any individuals who are infected have been identified (Supplementary Information section VIII).

Given $p$, the probability that $k$ members of a group of size $N$ are infected is described by a Poisson distribution with mean $\lambda=p N$. For $\lambda$ well below unity, the probability decreases rapidly with increasing $k$. At very low $p$, the optimal $N$ is very large, so $D=\log _{L}[N] \gg 1$. The advantages of the hypercube algorithm are particularly clear in this limit. Therefore, we describe this limit first before discussing realistic values of $D$. In this section we asssume, for simplicity, perfect accuracy of all tests.

The first round of slice tests-as described above-yields, for $L=3$, a set of triples of zeros and ones, that is, $\{1,0,0\},\{1,1,0\}$ or $\{1,1,1\}$ or a permutation thereof, for every principal direction of the lattice. Let $\sigma$ be the sum of the three values (so $\sigma=1,2$ or 3 ) and $d_{\sigma}$ the number of directions in which the value $\sigma$ occurs, so $d_{1}+d_{2}+d_{3}=D$. For $D \gg 1$, the number of group members who are infected $(k)$ may be accurately inferred from the observed values of $d_{\sigma}$, even before any individuals who are infected are identified. Knowing $k$, we then find all individuals who are infected as follows. First, if $k=1$, then $d_{1}=D$. Each positive slice indicates the coordinate value in that direction. Thus, the individual who is infected is identified in one round of slice tests. Second, suppose $k=2$, then $d_{2}>0$ but $d_{3}=0$. If $d_{2}=1$, the two individuals who are infected are immediately identified. If $d_{2}>1$, choose one of the directions with $\sigma=2$, and treat the two positive slices as smaller hypercubes, each containing one individual who is infected. A further round of slice tests identifies one and the other is found by elimination. Third, if $k=3$ then, at large $D$, at least one direction has $\sigma=3$. Choose one such direction and treat two of the positive slices as smaller hypercubes, each containing one individual who is infected. A slice test on each identifies two individuals who are infected and the third is found by elimination; if $k>3$, the number of rounds of slice tests required to identify all individuals who are infected is slightly larger than $k$. However, for the optimal value of group size, the probability to have $k>3$ members who are infected is negligibly small.

Thus, in the large $D$ limit, to a good approximation $k$ rounds of slice tests suffice to identify $k$ individuals who are infected. In the Supplementary Information, we show that, at low prevalence $p$, assuming Poisson statistics, the expected number of tests per person $\langle T\rangle / N$ that is required to identify all individuals who are infected is minimized for $N \approx 0.350 / p$. At this optimal group size, $\langle T\rangle / N \approx e p \ln (0.734 / p)$ (Fig. 3). The reciprocal of this number is the efficiency gain-that is, the cost savings factor-relative to testing every individual.

For practical applications, we are interested in the efficiency of the algorithm at modest values of $D$ such as 3,4 or 5 . This requires a more intricate analysis, the details of which we provide in the Supplementary

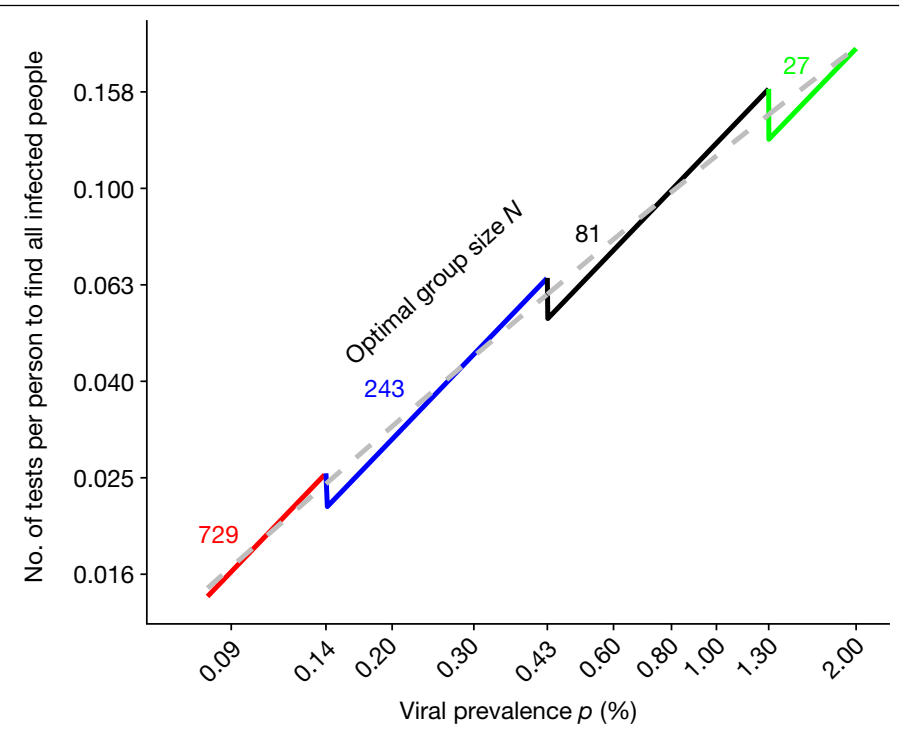

Fig. 3 | Expected number of tests per person, to find all individuals who are infected, using the hypercube algorithm. The results are shown on a log-log plot. The dashed grey line shows ep $\ln (0.734 / p)$, the result obtained in the large $D$ approximation, for which the optimal group size $N \approx 0.35 / p$. The coloured lines show the results obtained from a detailed analysis when the group size $N=3^{D}$ with $D$ an integer. Where $0.35 / p$ is an exact power of 3 , as at the left end of each coloured curve, optimal performance is attained. As $p$ is increased, a growing fraction of sites in the $3^{D}$ hypercube are left empty, until $0.35 / p$ is again an exact power of 3 (Supplementary Information).

Information. However, some simple and general statements are included here. First, when all directions yield $\sigma=1$, only one individual is infected and they are immediately and uniquely identified. This is the most probable outcome of the first round of slice tests. Second, when $\sigma>1$ in only one direction then two (or three) individuals who are infected are uniquely identified without further tests. If $\sigma>1$ in more than one direction, a second round of slice tests is needed. We can eliminate any slice that tested negative in the first round of slice tests and thus work with a smaller hypercube. We make only one approximation in our analysis, namely we assume the infected samples are rare in the hypercube. They may then be treated as independent, randomly chosen points. Within this approximation, we compute the probabilities through to the second round of slice tests. Notably, we find that the hypercube algorithm remains highly efficient at modest values of $D$. For example, for $\lambda=0.35$ and $D=4$, in $93.3 \%$ of cases one round of slice tests suffices to identify all individuals who are infected. For the remaining $6.7 \%$ of cases, one more round suffices in all but $0.01 \%$ of cases, a very low theoretical failure rate (which, we emphasize, does not include experimental errors). The expected total number of tests per person, for $D=3,4$ and 5 , is plotted in Fig. 3. When $0.35 / p$ is an exact power of 3, as is the case at the left end of each coloured curve in Fig. 3, the performance is best relative to the large $D$ formula given in the previous paragraph. As $p$ is increased, an increasing fraction of sites in the $3^{D}$ hypercube are left empty until the next exact power of 3 is reached. Nevertheless, at the values of $p$ shown, pooling always results in a high efficiency gain. As Fig. 3 shows, the large $D$ approximation provides a surprisingly good (and very convenient) fit to the low $D$ results (further details are provided in Supplementary Information sections IV-VII).

\section{Largely parallel searches are preferred}

Some search methods require fewer tests but more rounds of testing. A binary search ${ }^{2,3}$, for example, finds one individual among $N$ in $\sim \log _{2}[N]$ tests, a factor of $\ln [2] \approx 1.88$ fewer tests than needed by our hypercube algorithm at large $D$. However, the tests must be performed serially, 
requiring $\log _{2}(1 / p)$ rounds of testing. For $p=0.4 \%$ (or $0.15 \%$ ) a binary search takes 8 (or more than 9 ) rounds of testing whereas a hypercube search takes typically 2 and occasionally 3 rounds (in both cases). For a highly infectious disease such as COVID-19, saving time is crucial because individuals who are infected and still at large can infect others. The doubling time for COVID-19 has been estimated at $\tau_{2} \approx 2$ days s $^{14,26}$. If each testing round takes $\tau$ days, the viral prevalence in the population at large grows by $\sim(1 / p)^{\tau / \tau_{2}}$ during a binary search. If this growth factor exceeds eln (2), a binary search will do worse than a hypercube search. Assuming $\tau \approx 1 / 3$ day, we find that for $p<1 \%$, the hypercube search is preferred. Another advantage of the hypercube search is that it includes many consistency checks. For example, finding $\sigma=1$ in one direction and $\sigma=0$ in the others indicates a testing error. By contrast, a binary search relies on repeated testing of the positive sample, so that a single false-negative result can prematurely terminate the search.

\section{Conclusions}

The hypercube algorithm offers an attractive compromise between minimizing the total number of tests to reduce costs and maximizing the speed of testing to reduce the spread of the virus. We have demonstrated its viability for group sizes up to 100 samples, showing that cost savings of a factor of nearly 20 can, in principle, already be achieved. We have quantified the loss of sensitivity due to dilution and discussed a number of ways in which it may be mitigated-for example, through frequently repeated group tests. These strategies could enable the use of larger pool sizes, bringing even greater cost savings at low prevalence. The most striking aspect of our approach is how rapidly the cost of testing a population can fall, pooled test sensitivity permitting, as the viral prevalence decreases. This should incentivise decision-makers to act firmly to drive the prevalence down through mass screening, contact tracing and isolation. As the viral prevalence is reduced, all aspects of this strategy become more and more affordable.

\section{Online content}

Any methods, additional references, Nature Research reporting summaries, source data, extended data, supplementary information, acknowledgements, peer review information; details of author contributions and competing interests; and statements of data and code availability are available at https://doi.org/10.1038/s41586-020-2885-5.

1. Dorfman, R. The detection of defective members of large populations. Ann. Math. Stat 14, 436-440 (1943).

2. Hwang, F. K. A method for detecting all defective members in a population by group testing. J. Am. Stat. Assoc. 67, 605-608 (1972).

3. Allemann, A. in Information Theory, Combinatorics, and Search Theory. Lecture Notes in Computer Science Vol. 7777 (Aydinian, H. et al.) 569-596 (Springer, 2013).

4. Aldridge, M., Johnson, O. \& Scarlett, J. Group testing: an information theory perspective. Found. Trends Commun. Inf. Theory 15, 196-392 (2019).

5. Ghosh, S. et al. A compressed sensing approach to group-testing for COVID-19 detection. Preprint at https://arxiv.org/abs/2005.07895 (2020).
6. Heng, B. W. \& Scarlett, J. Non-adaptive group testing in the linear regime: strong converse and approximate recovery. Preprint at https://arxiv.org/abs/2006.01325 (2020).

7. Broder, A. Z. \& Kumar, R. A note on double pooling tests. Preprint at https://arxiv.org/ abs/2004.01684 (2020).

8. Arevalo-Rodriguez, l. et al. False negative results of initial RT-PCR assays for COVID-19: a systematic review. Preprint at https://doi.org/10.1101/2020.04.16.20066787 (2020).

9. Lohse, S. et al. Pooling of samples for testing for SARS-CoV-2 in asymptomatic people. Lancet Infect. Dis. 20, 1231-1232 (2020).

10. Yelin, I. et al. Evaluation of COVID-19 RT-qPCR test in multi sample pools. Clin. Infect. Dis. https://doi.org/10.1093/cid/ciaa531 (2020).

11. Shental, N. et al. Efficient high-throughput SARS-CoV-2 testing to detect asymptomatic carriers. Sci. Adv. 6, eabc5961 (2020).

12. Medicare. Medicare Administrative Contractor (MAC) COVID-19 Test Pricing. https://www. cms.gov/files/document/mac-covid-19-test-pricing.pdf (2020).

13. Corman, V. M. et al. Detection of 2019 novel coronavirus (2019-nCoV) by real-time RT-PCR. Euro Surveill. 25, 2000045 (2020).

14. Sanche, S. et al. High contagiousness and rapid spread of severe acute respiratory syndrome coronavirus 2. Emerg. Infect. Dis. 26, 1470-1477 (2020).

15. Office of National Statistics. Coronavirus (COVID-19) Infection Survey pilot: England and Wales, 4 September 2020. https://www.ons.gov.uk/peoplepopulationandcommunity/ healthandsocialcare/conditionsanddiseases/bulletins/ coronaviruscovid19infectionsurveypilot/englandandwales4september2020 (UK Department of Health \& Social Care, 2020).

16. Pouwels, K. B. et al. Community prevalence of SARS-CoV-2 in England: results from the ONS Coronavirus Infection Survey Pilot. Preprint at https://doi.org/10.1101/ 2020.07.06.20147348 (2020).

17. Office of National Statistics. COVID-19 Infection Survey (Pilot): Methods and Further Information: Test Sensitivity and Specificity. https://www.ons.gov.uk/ peoplepopulationandcommunity/healthandsocialcare/conditionsanddiseases/ methodologies/covid19infectionsurveypilotmethodsandfurtherinformation\#test-sensitivi ty-and-specificity (UK Department of Health \& Social Care, 2020).

18. Office of National Statistics. Weekly Statistics for NHS Test and Trace (England) and coronavirus testing (UK): 27 August to 2 September. Annex Table 1: People newly tested for COVID-19 under Pillars 1 and 2, England. https://www.gov.uk/government/ publications/nhs-test-and-trace-england-and-coronavirus-testing-uk-statistics-27august-to-2-september-2020/weekly-statistics-for-nhs-test-and-trace-englandand-coronavirus-testing-uk-27-august-to-2-september\#annex-table-1 (UK Department of Health \& Social Care, 2020).

19. Reijns M. A. M. et al. A sensitive and affordable multiplex RT-qPCR assay for SARS-CoV-2 detection. Preprint at https://doi.org/10.1101/2020.07.14.20154005 (2020).

20. Wölfel, R. et al. Virological assessment of hospitalized patients with COVID-2019. Nature 581, 465-469 (2020).

21. Long, Q. X. et al. Clinical and immunological assessment of asymptomatic SARS-CoV-2 infections. Nat. Med. 26, 1200-1204 (2020).

22. Watson, J., Whiting, P. F. \& Brush, J. E. Interpreting a covid-19 test result. Br. Med. J. 369, $\mathrm{m} 1808$ (2020)

23. Kucirka, L. M., Lauer, S. A., Laeyendecker, O., Boon, D. \& Lessler, J. Variation in false-negative rate of reverse transcriptase polymerase chain reaction-based SARS-CoV-2 by time since exposure. Ann. Intern. Med. 173, 262-267 (2020)

24. Jaafar, R. et al. Correlation between $3790 \mathrm{qPCR}$ positives samples and positive cell cultures including 1941 SARS-CoV-2 isolates. Clin. Infect. Dis. https://doi.org/10.1093/cid/ ciaa1491 (2020).

25. Service, R. F. One number could help reveal how infectious a COVID-19 patient is. Should test results include it? Science https://www.sciencemag.org/news/2020/09/one-numbercould-help-reveal-how-infectious-covid-19-patient-should-test-results (2020).

26. Afshordi, N., Holder, B., Bahrami, M. \& Lichtblau, D. Diverse local epidemics reveal the distinct effects of population density, demographics, climate, depletion of susceptibles, and intervention in the first wave of covid-19 in the United States. Preprint at https://arxiv. org/abs/2007.00159 (2020).

Publisher's note Springer Nature remains neutral with regard to jurisdictional claims in published maps and institutional affiliations.

(c) The Author(s), under exclusive licence to Springer Nature Limited 2020, corrected publication 2021 


\section{Methods}

\section{Observational study design}

We conducted an experiment to evaluate the hypothesis that known SARS-CoV-2-positive oropharyngeal swab specimens collected during COVID-19 surveillance in Rwanda will test positive after they are combined with as many as 99 known SARS-CoV-2-negative specimens. This was followed by an observational study that aimed to apply our hypercube algorithm to increase the efficiency of community testing for COVID-19 in Rwanda. In the experiment, two different sets of sample pools were tested for SARS-CoV-2 using RT-PCR. Each set consisted of three sample pools that contained one known SARS-CoV-2-positive sample diluted in ratios of $1: 20,1: 50$ and $1: 100$ by combining it with equivalent amounts of 19,49 and 99 known SARS-CoV-2-negative samples, respectively (Fig. 2 and Extended Data Table 2). In the observational study, 1,280 individuals selected from the community were tested for SARS-CoV-2 using RT-PCR. One third of the individuals were participants in a screening for severe acute respiratory infections and influenza-like illness conducted in $30 \%$ of the health facilities found across the 30 districts of Rwanda. The remaining two thirds were from COVID-19 screening of at-risk groups in the capital city of Kigali. The latter group consisted mainly of individuals (market vendors, bank agents and supermarket agents) who remained active during the lockdown imposed by the Government of Rwanda to contain COVID-19. Extended Data Table 1 summarizes the characteristics of the study participants.

The positive fraction of RT-PCR tests for SARS-CoV-2 conducted in Rwanda in March 2020 suggests an upper bound of $2 \%$ for the virus prevalence in the country. Using $p=2 \%$ in the hypercube algorithm indicated an optimal sample group size of 17.5. For convenience, the 1,280 individual samples were combined in 64 groups of 20 samples before testing for SARS-CoV-2 (Extended Data Fig. 1).

We used two established experimental protocols for SARS-CoV-2 testing. The first is a protocol by DAAN Gene and Sun Yat-sen University that is available online (https://prolabcorp.com/daanrt-pcr-reagent-set-for-covid-19-real-time-detection-for-48-samplesresearch-use-only), and is also under review by the WHO. The second protocol ${ }^{13}$ is widely used by the scientific community. The first protocol is used for routine screening for SARS-CoV-2, whereas the second protocol is used only if the first protocol produces a positive result and confirmation is therefore required.

\section{Sample collection and pool design}

Oropharyngeal swabs were collected by wiping the tonsils and posterior pharynx wall with two swabs, and the swab heads were immersed in $3 \mathrm{ml}$ viral transport medium. Samples were transported in viral transport medium to the Rwanda National Reference Laboratory immediately after collection. Samples that had to be transported over a long distance were stored in dry ice. Each sample had a volume of $3 \mathrm{ml}$, of which an aliquot of $200 \mu \mathrm{l}$ was used for pooled testing, and the remainder was temporarily stored at $-20^{\circ} \mathrm{C}$ until the result of the pooled testing was known. The aliquot $(200 \mu \mathrm{l})$ of each sample was mixed with aliquots with the same volume of other samples of the same pool in a Falcon $15-\mathrm{ml}$ conical tube and, after vortexing for $5 \mathrm{~s}, 200 \mu \mathrm{l}$ of the mixture was pipetted for downstream RNA extraction. Then, $5 \mu \mathrm{l}$ of the extracted RNA was added to $20 \mu \mathrm{l}$ of master mix for a total of $25 \mu \mathrm{l}$ to be amplified by RT-PCR. If a pool tested positive, stored samples from that pool were processed to identify the positive samples. Individual samples were barcoded, making it easy to trace individuals that tested positive and minimizing the risk of confusion of samples. Pool design and subsequent experimental analysis (see 'RT-PCR for SARS-CoV-2') were implemented with the aid of a robot to reduce human error.

\section{RT-PCR for SARS-CoV-2}

Total viral RNA was extracted from swab specimens using the QIAamp Viral RNA 91 Mini Kit (QIAGEN), according to the manufacturer's instructions. RNA samples were screened for SARS-CoV-2 using a 2019-nCoV RNA RT-PCR test that targets two genes that, respectively, encode an open reading frame (denoted orflab) and a nucleocapsid protein (denoted $N$ ) (DAAN Gene and Sun Yat-sen University). For orf1ab, CCCTGTGGGTTTTACACTTAA and ACGATTGTGCATCAGCTGA were used as forward and reverse primers, respectively, together with a 5'-VIC-CCGTCTGCGGTATGTGGAAAGGTTATGG-BHQ1-3' probe. For $N$, GGGGAACTTCTCCTGCTAGAAT and CAGACATTTTGCTCTCAAGCTG were used as forward and reverse primers, respectively, together with a 5'-FAM-TTGCTGCTGCTTGACAGATT-TAMRA-3' probe. The RT-PCR reaction was set up according to the manufacturer's protocol, with a total volume of $25 \mu \mathrm{l}$. The reaction was run on the ABI Prism 7500 SDS Instrument (Applied Biosystems) at $50^{\circ} \mathrm{C}$ for $15 \mathrm{~min}$ for reverse transcription, denatured at $95^{\circ} \mathrm{C}$ for $15 \mathrm{~min}$, followed by 45 PCR cycles of $94^{\circ} \mathrm{C}$ for $15 \mathrm{~s}$ and $55^{\circ} \mathrm{C}$ for $45 \mathrm{~s}$. A threshold cycle $\left(C_{\mathrm{t}}\right) \leq 40$ indicated a positive test; $C_{\mathrm{t}}>40$ indicated a negative test. Positive controls for the reaction showed amplification as determined by curves for FAMand VIC-detection channels, and $C_{\mathrm{t}} \leq 32$. Positive tests were confirmed using LightMix SarbecoV E-gene and LightMix Modular SARS-CoV-2 RdRp RT-PCR tests that target the envelope $(E)$ and RNA-directed RNA polymerase ( $r d r p$ ) genes, respectively, as described by the manufacturer (TIB MOLBIOL). Both the primers used and the RT-PCR reaction conditions were previously described ${ }^{13}$.

\section{Statistical analysis}

$C_{\mathrm{t}}$ values were tested for normality using the Shapiro-Wilk test. A confidence bound for a sample of $n C_{\mathrm{t}}$ values was calculated as $\bar{C}_{\mathrm{t}} \pm t_{\mathrm{df}}^{*} \times s$, where $\bar{C}_{\mathrm{t}}$ is the sample mean, $s$ is the sample standard error of the mean and $t_{\mathrm{d} . \mathrm{f}}^{*}$ is an appropriate quantile of the Student's $t$-distribution with $n$-1 degrees of freedom (d.f.). A confidence bound for the sum of the means of two samples of $C_{\mathrm{t}}$ values of sizes $n_{1}$ and $n_{2}$, respectively, was calculated using the same formula, with $\bar{C}_{\mathrm{t}}$ set to the sum of the individual sample means, $s$ set to the sum of the standard errors of the individual sample means and d.f. set to the smaller of $n_{1}-1$ and $n_{2}-1$. Statistical analysis was done using the R statistical computing environment (https://www.r-project.org/).

\section{Loss of sensitivity due to dilution}

To estimate the postdilution sensitivities of RT-PCR tests with different maximum numbers of PCR cycles, we combined two datasets. First, we used the mean and standard deviations of the number of additional PCR cycles required for a positive detection, after a $k$-fold dilution of a positive specimen (Fig. 2, showing the data in Extended Data Table 3). Second, we used the mean and standard deviation of $C_{\mathrm{t}}$ values for positive specimens sampled from a target population. We combined (or, more accurately, convolved) the two probability distributions, represented as Gaussians to calculate the sensitivity of a $\leq x$ cycle PCR test as the probability that the $C_{\mathrm{t}}$ value of a $k$-fold diluted positive specimen sampled from the same population will be $\leq x$. Using a representative sample of 33 positive specimens identified during clinical screening for SARS-CoV-2 in Rwanda (Extended Data Table 3), we estimate that a $\leq 40$-cycle PCR test targeting the SARS-CoV-2 $N$ (or orf1 $a b$ ) gene, respectively, has postdilution sensitivities for 20-, 50 - and 100 -fold dilutions of $95 \%, 92 \%$ and $89 \%$ (or $86 \%, 82 \%$ and $77 \%$, respectively). For a $\leq 44$-cycle PCR test targeting the $N$ (or orf1 $1 a b$ ) gene, we obtain postdilution sensitivities for 20-,50- and 100 -fold dilutions of $99 \%, 98 \%$ and $98 \%$ (or $96 \%, 94$ and $92 \%$ ), respectively. As mentioned in the paper, a maximum of 44 PCR cycles was used in the recent study of pooled testing for SARS-CoV-2 in Israel ${ }^{10}$.

As further checks, we applied the same analysis to (1) an independent sample of 107 positive specimens collected in Rwanda and (2) the previously published datase ${ }^{10}$, which consists of the 26 positive specimens identified in the previous study ${ }^{10}$. From the Rwandan dataset, we estimated that a 40-cycle PCR test targeting the $N$ (or orf1 $a b$ ) gene, respectively, has postdilution sensitivities for 20-, 50- and 100-fold 


\section{Article}

dilutions of $91 \%, 88 \%$ and $85 \%$ (or $85 \%, 81$ and $77 \%$, respectively). For a 44-cycle PCR test targeting the $N$ (or orf1ab) gene, respectively, the predicted sensitivities are $97 \%, 96 \%$ and $95 \%$ (or $94 \%, 92$ and $90 \%$ ). The previously published datase ${ }^{10}$ contains $C_{\mathrm{t}}$ values for only one genethe $E$ gene of SARS-CoV-2. On the basis of the arguments described above, we assume for simplicity that diluting a positive specimen by 20-, 50- and 100-fold adds approximately 5, 6 and 7, respectively, to the original $C_{\mathrm{t}}$ value. Applying these assumptions to the previously published dataset ${ }^{10}$, we infer postdilution sensitivities for $20-, 50$ - and 100 -fold dilutions of $94 \%, 92 \%$ and $89 \%$, respectively, for a $\leq 40$-cycle PCR test, and $99 \%, 98 \%$ and $97 \%$, for a $\leq 44$-cycle PCR test. These results are comparable to those reported from our experiments. Together, these findings confirm that diluting positive samples does result in a loss of sensitivity, but that much of the loss can be offset by increasing the number of PCR cycles. In particular, sensitivities above $90 \%$ can be achieved for 100 -fold dilution by using 44 PCR cycles, only $10 \%$ more than the number routinely employed.

\section{Ethics approval}

Ethics approval was obtained from the Rwanda National Ethics Committee (FWA Assurance No. 00001973 IRB 00001497 of IORG0001100/20March2020) and written informed consent was obtained from the participants.

\section{Reporting summary}

Further information on research design is available in the Nature Research Reporting Summary linked to this paper.

\section{Data availability}

All data that support the findings of this study are available from the corresponding authors upon reasonable request.

\section{Code availability}

All codes that were used in the study are available from the corresponding authors upon reasonable request.

Acknowledgements We thank the Rwanda Ministry of Health through RBC for discussions and correspondence, K. Smith and C. Squire for providing encouragement and references, and A. Jackson for discussions. Research at AIMS is supported in part by the Carnegie Corporation of New York and by the Government of Canada through the International Development Research Centre and Global Affairs Canada. Research at the Perimeter Institute is supported in part by the Government of Canada through the Department of Innovation, Science and Economic Development Canada and by the Province of Ontario through the Ministry of Colleges and Universities. The data collection, SARS-CoV-2 molecular experiments and analysis of the study were supported by the Government of Rwanda (Rwanda Biomedical Centre/Ministry of Health) and the Académie de Recherche et d'Enseignement Supérieur in collaboration with the University of Rwanda (ARES-UR Programme). All statistical and mathematical analyses were supported by the African Institute for Mathematical Sciences (AIMS). The funders had no role in study design, data collection and analysis, decision to publish, or preparation of the manuscript.

Author contributions L.M. and S.N. coordinated the experiments; P.N., T.N., E.N., M.S., C.M. D.N. and M.N. contributed to patient recruitment and data collection from the community; P.N., Y.B., J.S., A.U., R.R., E.L.N., E.M., N.R., I.E.M., J.B.M., C.M.M. and E.U. contributed to laboratory RT-PCR test validation, data analysis and interpretation. W.N. and N.T. contributed to the theory.

Competing interests The authors declare no competing interests.

Additional information Supplementary information is available for this paper at https://doi.org/10.1038/s41586-0202885-5.

Correspondence and requests for materials should be addressed to N.T. or W.N. Peer review information Nature thanks Maimuna Majumder, Sigrun Smola and the other, anonymous, reviewer(s) for their contribution to the peer review of this work. Peer reviewer reports are available.

Reprints and permissions information is available at http://www.nature.com/reprints. 


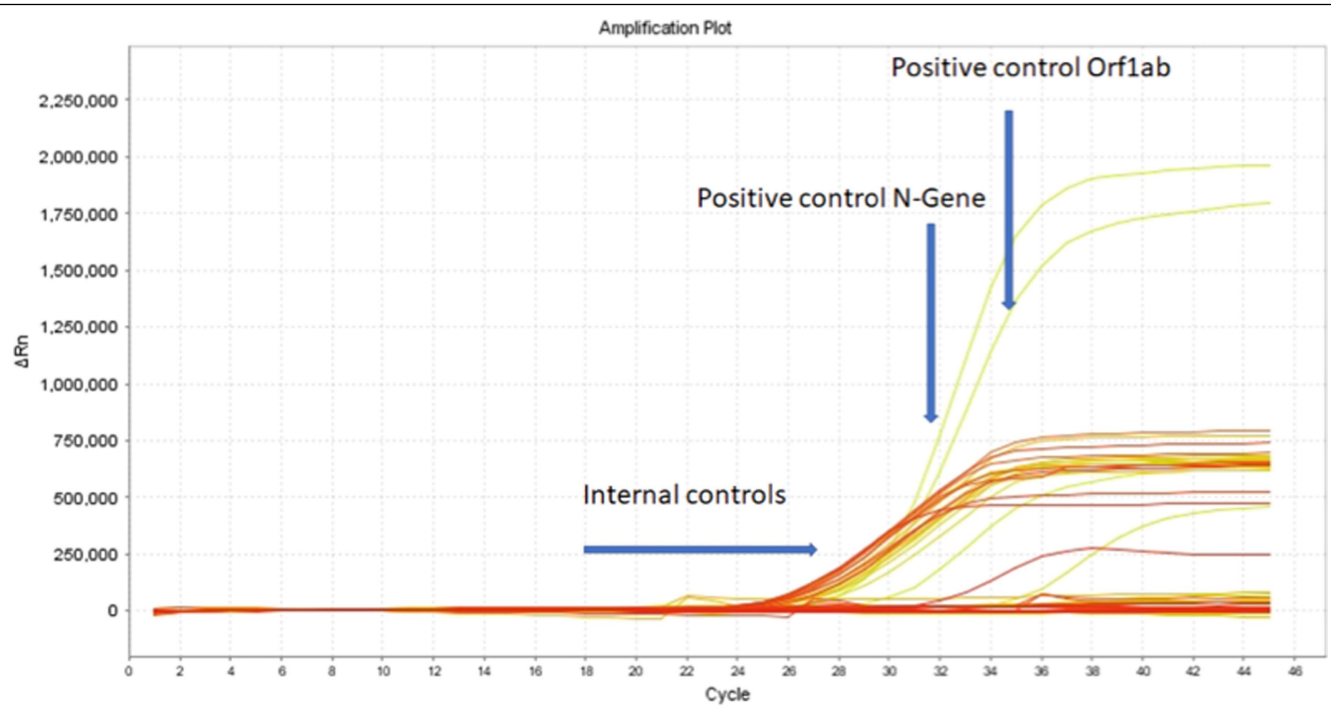

Extended Data Fig. 1 | Amplification plot for sample pools. Each of the 64 sample pools described in the text tests negative for SARS-CoV-2: the RT-PCR fluorescence curves show below-threshold net fluorescence values. By contrast, for both target genes of the positive control, the fluorescence curves cross the threshold after $32 \mathrm{PCR}$ cycles. $\triangle \mathrm{Rn}$ denotes the difference between the fluorescence signal generated by a sample and a baseline signal. The yellow curves reaching $\Delta \mathrm{Rn} \approx 2,000,000$ and $1,750,000$ represent the positive control for the $N$ and orf1abgenes, respectively. The other yellow and orange curves represent internal controls. 


\section{Article}

Extended Data Table 1 | Characteristics of participants of the field trial for the hypercube algorithm in Rwanda

\begin{tabular}{lrrrrr}
\hline Characteristics & Female & \multicolumn{3}{c}{ Male } & \\
\hline \hline Cluster & $\mathbf{n}$ & $\mathbf{\%}$ & $\mathbf{n}$ & $\mathbf{\%}$ & $\mathbf{n}$ \\
At-risk & 327 & 38.3 & 526 & 61.7 & 853 \\
SARI/ILI* & 190 & 44.5 & 237 & 55.5 & 427 \\
Total & 517 & 40.4 & 763 & 59.6 & 1,280 \\
Age group & & & & & \\
$0-14$ & 62 & 55.9 & 49 & 44.1 & 111 \\
$15-34$ & 263 & 40.2 & 392 & 59.8 & 655 \\
$35-49$ & 147 & 38.7 & 233 & 61.3 & 380 \\
$50-64$ & 30 & 28.0 & 77 & 72.0 & 107 \\
$65+$ & 15 & 55.6 & 12 & 44.4 & 27 \\
Total & 517 & 40.4 & 763 & 59.6 & 1,280 \\
\hline
\end{tabular}

Mean age of participants (sd) 31.97 (14.27)

For more information, see Methods, 'Observational study design'.

*SARI, severe acute respiratory infections; ILI, influenza-like illness. 


\section{Extended Data Table 2 | Positive specimens are detected after strong dilution}

\begin{tabular}{|c|c|c|c|c|c|}
\hline & ID & $\mathrm{N}$-gene & Orf1ab & E-gene & RdRp \\
\hline \multirow[t]{4}{*}{1} & B16121 & 21.7 & 22.9 & 24.5 & 28 \\
\hline & B16121-20 & 29 & 31 & & \\
\hline & B16121-50 & 29.74 & 30.51 & & \\
\hline & B16121-100 & 30.54 & 30.95 & & \\
\hline \multirow[t]{4}{*}{2} & B16122 & 25.3 & 26.7 & 28.3 & 32 \\
\hline & B16122-20 & 26.81 & 28.5 & & \\
\hline & B16122-50 & 27.75 & 29.4 & & \\
\hline & B16122-100 & 29.07 & 30.2 & & \\
\hline \multirow[t]{4}{*}{3} & B09495 & 28.2 & 31.2 & 27 & 29.7 \\
\hline & B09495-20 & 33.2 & 36.9 & & \\
\hline & B09495-50 & 34.6 & 38.2 & & \\
\hline & B09495-100 & 35.4 & 39 & & \\
\hline \multirow[t]{4}{*}{4} & D34141 & 26.9 & 29.1 & 24 & 25.6 \\
\hline & D34141-20 & 28.9 & 32.2 & & \\
\hline & D34141-50 & 30.2 & 33 & & \\
\hline & D34141-100 & 31 & 34.3 & & \\
\hline \multirow[t]{4}{*}{5} & D36394 & 29.7 & 31.1 & 30.5 & 31.9 \\
\hline & D36394-20 & 33.62 & 36.52 & & \\
\hline & D36394-50 & 34.85 & 37.67 & & \\
\hline & D36394-100 & 36.49 & 39.25 & & \\
\hline \multirow[t]{4}{*}{6} & D48370 & 25.9 & 27.2 & 26 & 26.4 \\
\hline & D48370-20 & 30.71 & 33.38 & & \\
\hline & D48370-50 & 32.1 & 34.8 & & \\
\hline & D48370-100 & 32.91 & 35.64 & & \\
\hline & & & & & \\
\hline
\end{tabular}

Six SARS-CoV-2-positive specimens that were detected during COVID-19 screening in Rwanda were analysed. The positive specimens were detected using a screening RT-PCR test targeting

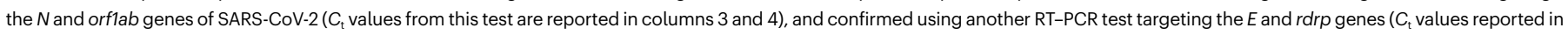

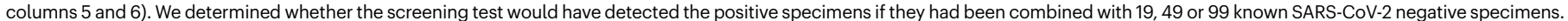

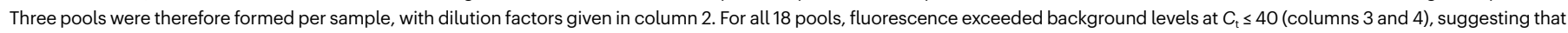
the positive samples would have been detected even if diluted 100 -fold. 
Extended Data Table 3 | Representative confirmed positive specimens detected during COVID-19 screening in Rwanda

\begin{tabular}{|c|c|c|c|c|c|c|c|c|c|c|}
\hline & ID & N-gene & CT & ORF ab1 & CT & E-gene & CT & RdRp & CT & Diagnosis \\
\hline 1 & D15943 & Positive & 17.2 & Positive & 18.5 & Positive & 18.1 & Positive & 19.2 & Positive \\
\hline 2 & B17418 & Positive & 19.33 & Positive & 22.26 & Positive & 16.87 & Positive & 18.38 & Positive \\
\hline 3 & B46255 & Positive & 20 & Positive & 21.1 & Positive & 20.1 & Positive & 20.8 & Positive \\
\hline 4 & B46255 & Positive & 20.1 & Positive & 21.1 & Positive & 20.1 & Positive & 20.8 & Positive \\
\hline 5 & 16121 & Positive & 21.7 & Positive & 22.9 & Positive & 24.5 & Positive & 28 & Positive \\
\hline 6 & C45273 & Positive & 22.78 & Positive & 24.38 & Positive & 22.85 & Positive & 23.55 & Positive \\
\hline 7 & D18543 & Positive & 23.1 & Positive & 24.2 & Positive & 23.1 & Positive & 24.2 & Positive \\
\hline 8 & B79243 & Positive & 23.2 & Positive & 24.1 & Positive & 23.3 & Positive & 23.3 & Positive \\
\hline 9 & B79243 & Positive & 23.2 & Positive & 24.1 & Positive & 23.3 & Positive & 23.3 & Positive \\
\hline 10 & D40438 & Positive & 23.29 & Positive & 26.68 & Positive & 24.47 & Positive & 25.73 & Positive \\
\hline 11 & B86037 & Positive & 24.64 & Positive & 27.14 & Positive & 21.3 & Positive & 23.25 & Positive \\
\hline 12 & D04800 & Positive & 25.2 & Positive & 26.9 & Positive & 24.8 & Positive & 27.1 & Positive \\
\hline 13 & D04800 & Positive & 25.2 & Positive & 26.9 & Positive & 24.8 & Positive & 27.1 & Positive \\
\hline 14 & D15947 & Positive & 25.3 & Positive & 26.4 & Positive & 25.6 & Positive & 27.1 & Positive \\
\hline 15 & 16122 & Positive & 25.3 & Positive & 26.7 & Positive & 28.3 & Positive & 32 & Positive \\
\hline 16 & D48370 & Positive & 25.9 & Positive & 27.2 & Positive & 26 & Positive & 26.4 & Positive \\
\hline 17 & B90541 & Positive & 26.3 & Positive & 29.6 & Positive & 27.2 & Positive & 27.8 & Positive \\
\hline 18 & CV000054088 & Positive & 26.6 & Positive & 27.6 & Positive & 24.9 & Positive & 27.8 & Positive \\
\hline 19 & D34141 & Positive & 26.9 & Positive & 29.1 & Positive & 24 & Positive & 25.6 & Positive \\
\hline 20 & CV000019058 & Positive & 27.7 & Positive & 28.7 & Positive & 26.2 & Positive & 28.8 & Positive \\
\hline 21 & B87522 & Positive & 27.82 & Positive & 30.64 & Positive & 25.75 & Positive & 26.63 & Positive \\
\hline 22 & B09495 & Positive & 28.2 & Positive & 31.2 & Positive & 27 & Positive & 29.7 & Positive \\
\hline 23 & D17033 & Positive & 28.8 & Positive & 31.8 & Positive & 28.9 & Positive & 29.7 & Positive \\
\hline 24 & D36394 & Positive & 29.7 & Positive & 31.1 & Positive & 30.5 & Positive & 31.9 & Positive \\
\hline 25 & B46304 & Positive & 31.6 & Positive & 36.2 & Positive & 36.3 & Positive & 36.7 & Positive \\
\hline 26 & D15317 & Positive & 31.7 & Positive & 34.6 & Positive & 33.1 & Positive & 33.7 & Positive \\
\hline 27 & B86032 & Positive & 32.57 & Positive & 35.12 & Positive & 30.79 & Positive & 31.57 & Positive \\
\hline 28 & B65096 & Positive & 33.3 & Positive & 35.5 & Positive & 32.1 & Positive & 34.9 & Positive \\
\hline 29 & D11510 & Positive & 33.5 & Positive & 36.3 & Positive & 33.7 & Positive & 34.7 & Positive \\
\hline 30 & B64423 & Positive & 33.54 & Positive & 36.69 & Positive & 34.15 & Positive & 36.19 & Positive \\
\hline 31 & B46275 & Positive & 34.1 & Positive & 35.9 & Positive & 34.1 & Positive & 35.4 & Positive \\
\hline 32 & B86031 & Positive & 35.03 & Positive & 37.47 & Positive & 33.5 & Positive & 34.29 & Positive \\
\hline 33 & B43708 & Positive & 38.12 & Positive & 39.7 & Positive & 34.94 & Positive & 35.81 & Positive \\
\hline
\end{tabular}

The specimens were detected using a screening RT-PCR test targeting the $N$ and orf1ab genes of SARS-CoV-2 ( $C_{t}$ values from this test are reported in columns 3 and 5$)$. The samples were subsequently confirmed as positive using another RT-PCR test targeting the $E$ and $r d r p$ genes $\left(C_{t}\right.$ values are reported in columns 7 and 9$)$. 


\section{Reporting Summary}

Nature Research wishes to improve the reproducibility of the work that we publish. This form provides structure for consistency and transparency in reporting. For further information on Nature Research policies, see our Editorial Policies and the Editorial Policy Checklist.

\section{Statistics}

For all statistical analyses, confirm that the following items are present in the figure legend, table legend, main text, or Methods section.

n/a Confirmed

$\square$ \The exact sample size $(n)$ for each experimental group/condition, given as a discrete number and unit of measurement

$\square$ \A statement on whether measurements were taken from distinct samples or whether the same sample was measured repeatedly

$\square$ The statistical test(s) used AND whether they are one- or two-sided

$\square$ Only common tests should be described solely by name; describe more complex techniques in the Methods section.

Х $\square$ A description of all covariates tested

Х $\square$ A description of any assumptions or corrections, such as tests of normality and adjustment for multiple comparisons

$\triangle$ A full description of the statistical parameters including central tendency (e.g. means) or other basic estimates (e.g. regression coefficient)

$\bigotimes$ AND variation (e.g. standard deviation) or associated estimates of uncertainty (e.g. confidence intervals)

Х For null hypothesis testing, the test statistic (e.g. $F, t, r$ ) with confidence intervals, effect sizes, degrees of freedom and $P$ value noted

Wive $P$ values as exact values whenever suitable.

Х $\square$ For Bayesian analysis, information on the choice of priors and Markov chain Monte Carlo settings

$\square$ \ For hierarchical and complex designs, identification of the appropriate level for tests and full reporting of outcomes

Х $\square$ Estimates of effect sizes (e.g. Cohen's $d$, Pearson's $r$ ), indicating how they were calculated

Our web collection on statistics for biologists contains articles on many of the points above.

\section{Software and code}

Policy information about availability of computer code

Data collection Raw data (Ct values) obtained from PCR-RT machines and built-in software available on request

Data analysis Open source code in R (V 4.02) (used to produce Figure 3) available on request; Mathematica ( $\mathrm{V} 12.1$ ) code used to obtain results in given in Supplementary Information available on request

For manuscripts utilizing custom algorithms or software that are central to the research but not yet described in published literature, software must be made available to editors and reviewers. We strongly encourage code deposition in a community repository (e.g. GitHub). See the Nature Research guidelines for submitting code \& software for further information.

\section{Data}

Policy information about availability of data

All manuscripts must include a data availability statement. This statement should provide the following information, where applicable:

- Accession codes, unique identifiers, or web links for publicly available datasets

- A list of figures that have associated raw data

- A description of any restrictions on data availability

All data and codes (both in $\mathrm{R}$ and in Mathematica) mentioned in the paper are available from the corresponding authors upon reasonable request 


\section{Field-specific reporting}

Please select the one below that is the best fit for your research. If you are not sure, read the appropriate sections before making your selection. \Life sciences

\section{Life sciences study design}

All studies must disclose on these points even when the disclosure is negative.

\section{Sample size}

1200 samples for proof of concept experiment on pooled testing, in discussion with Rwandan Ministry of Health; 140 samples for sensitivity analysis for positive samples, this was the largest sample available.

Data exclusions

None

Replication

Randomization

Blinding
Test sensitivity analysis (Section III of the paper) on 33 positive samples, on the basis of data presented in Methods Table 7, confirmed by analysis of 107 further samples as well as data taken from an independent study (Ref. 14)

\section{Reporting for specific materials, systems and methods}

We require information from authors about some types of materials, experimental systems and methods used in many studies. Here, indicate whether each material, system or method listed is relevant to your study. If you are not sure if a list item applies to your research, read the appropriate section before selecting a response.

Materials \& experimental systems

\begin{tabular}{l|l}
\hline$n / a$ & Involved in the study \\
$\square$ & $\square$ Antibodies \\
\hline & $\square$ Eukaryotic cell lines \\
$\square$ & $\square$ Palaeontology and archaeology \\
$\square$ & $\square$ Animals and other organisms \\
$\square$ & $\square$ Clinical data \\
$\square$ & $\square$ Dual use research of concern
\end{tabular}

\section{Human research participants}

Policy information about studies involving human research participants

Population characteristics

In Rwanda, 1280 individuals selected from the community were tested for SARS-CoV-2 using RT-PCR. One third of the individuals were participants in a screening for Severe Acute Respiratory Infections (SARI) and Influenza Like IIIness (ILI) conducted in 30 per cent of the health facilities found across the 30 districts of Rwanda. The remaining two thirds were from COVID-19 screening of at-risk groups in the capital city of Kigali. The latter group is comprised mainly of people (market vendors, bank agents, and supermarket agents) who remained active during the lockdown imposed by the Government of Rwanda to contain COVID-19. Table 5 summarises the characteristics of the study participants.

Recruitment Recruited by the Rwandan Ministry of Health COVID-19 team

Ethics oversight

Rwanda National Ethics Committee (Ref: FWA Assurance No. 00001973 IRB 00001497 of IORG0001100/20March2020). 


\section{Data deposition}

$\square$ Confirm that both raw and final processed data have been deposited in a public database such as GEO.

$\square$ Confirm that you have deposited or provided access to graph files (e.g. BED files) for the called peaks.

Data access links

May remain private before publication

Files in database submission

Genome browser session (e.g. UCSC)

Methodology

Replicates

Sequencing depth

Antibodies

Peak calling parameters

Data quality

Software used.
For "Initial submission" or "Revised version" documents, provide reviewer access links. For your "Final submission" document, provide a link to the deposited data.

Provide a list of all files available in the database submission.

Provide a link to an anonymized genome browser session for "Initial submission" and "Revised version" documents only, to enable peer review. Write "no longer applicable" for "Final submission" documents.

Describe the antibodies used for the ChIP-seq experiments; as applicable, provide supplier name, catalog number, clone name, and lot number.

Specify the command line program and parameters used for read mapping and peak calling, including the ChIP, control and index files

Describe the methods used to ensure data quality in full detail, including how many peaks are at FDR 5\% and above 5-fold enrichment.

Describe the software used to collect and analyze the ChIP-seq data. For custom code that has been deposited into a community repository, provide accession details.

\section{Flow Cytometry}

Plots

Confirm that:

$\square$ The axis labels state the marker and fluorochrome used (e.g. CD4-FITC).

$\square$ The axis scales are clearly visible. Include numbers along axes only for bottom left plot of group (a 'group' is an analysis of identical markers).

$\square$ All plots are contour plots with outliers or pseudocolor plots.

$\square$ A numerical value for number of cells or percentage (with statistics) is provided.

\section{Methodology}

Sample preparation

Instrument

Software

Cell population abundance

Gating strategy
Describe the sample preparation, detailing the biological source of the cells and any tissue processing steps used.

Identify the instrument used for data collection, specifying make and model number.

Describe the software used to collect and analyze the flow cytometry data. For custom code that has been deposited into community repository, provide accession details.

Describe the abundance of the relevant cell populations within post-sort fractions, providing details on the purity of the samples and how it was determined.

Describe the gating strategy used for all relevant experiments, specifying the preliminary FSC/SSC gates of the starting cell population, indicating where boundaries between "positive" and "negative" staining cell populations are defined.

Tick this box to confirm that a figure exemplifying the gating strategy is provided in the Supplementary Information.

\section{Magnetic resonance imaging}

Experimental design

Design type

Indicate task or resting state; event-related or block design 
Design specifications

Behavioral performance measures or block (if trials are blocked) and interval between trials.

State number and/or type of variables recorded (e.g. correct button press, response time) and what statistics were used to establish that the subjects were performing the task as expected (e.g. mean, range, and/or standard deviation across subjects).
Acquisition

Imaging type(s)

Field strength

Sequence \& imaging parameters

Area of acquisition

Diffusion MRI

Used

\section{Preprocessing}

Preprocessing software

Normalization

Normalization template

Noise and artifact removal

Volume censoring
Specify: functional, structural, diffusion, perfusion.

Specify in Tesla

Specify the pulse sequence type (gradient echo, spin echo, etc.), imaging type (EPI, spiral, etc.), field of view, matrix size, slice thickness, orientation and TE/TR/flip angle.

State whether a whole brain scan was used OR define the area of acquisition, describing how the region was determined.

\section{Statistical modeling \& inference}

Model type and settings

Specify type (mass univariate, multivariate, RSA, predictive, etc.) and describe essential details of the model at the first and second levels (e.g. fixed, random or mixed effects; drift or auto-correlation).

Effect(s) tested

Define precise effect in terms of the task or stimulus conditions instead of psychological concepts and indicate whether ANOVA or factorial designs were used.

Specify type of analysis:

$\square$ Whole brain $\square$ ROl-based $\square$ Both

Statistic type for inference (See Eklund et al. 2016)

Specify voxel-wise or cluster-wise and report all relevant parameters for cluster-wise methods.

Correction

Models \& analysis

$\mathrm{n} / \mathrm{a}$ | Involved in the study

Х Functional and/or effective connectivity

Х Graph analysis

Х $\square$ Multivariate modeling or predictive analysis 